Pacific Journal of Mathematics

DIFFERENTIABILITY OF MINIMAL SURFACES AT THE 


\section{DIFFERENTIABILITY OF MINIMAL SURFACES AT THE BOUNDARY}

\section{Frank DAVID LesLey}

Let $\Gamma$ be a Jordan curve in $R^{3}$ and $F(z)=(u(z), v(z), w(z))$ : $\{|z| \leqq 1\} \rightarrow R^{3}$ be a solution of Plateau's problem for $\Gamma$, where $z=x+i y$ are isothermal parameters. Then $u, v, w$ are harmonic in $\{|z|<1\}$ and are the real parts of analytic functions $\lambda, \mu, \nu$. Using the Poisson integral and the defining properties of minimal surfaces, Kellogg's theorem for conformal mapping is generalized by proving: 1. If $\Gamma \in C^{1, \alpha}$, $0<\alpha<1$, then $\lambda, \mu, \nu \in C^{1, \alpha}$ for $|z| \leqq 1$ and if $\Gamma \in{ }^{1,1}$ then $\lambda^{\prime}, \mu^{\prime}, \nu^{\prime}$ have modulus of continuity $K t \log 1 / t$ for $|z| \leqq 1 ; K$ and the Holder constants depend only on the geometry of $\Gamma$. 2. If $\Gamma \in C^{n, \omega(t)}, n \geqq 2$, where $\omega(t)$ is a modulus of continuity satisfying a Dini condition, then $\lambda, \mu, \nu \in C^{n, \omega^{*}(t)}$ for $|z| \leqq 1$, where $\omega^{*}(t)$ is a certain modulus of continuity. Once again $\omega^{*}$ depends only on $\Gamma$.

Let $\Gamma$ be a closed Jordan curve in $R^{3}$. Then $S$ is called a generalized minimal surface spanning $\Gamma$ if $S$ is represented by a triple of real valued functions

$$
F(z)=(u(z), v(z), w(z)):\{|z| \leqq 1\} \rightarrow R^{3} \quad\left(z=x+i y=r e^{i \theta}\right)
$$

such that

(a) $u, v, w$ are harmonic in $|z|<1$ and continuous in $|z| \leqq 1$

(b) $x$ and $y$ are isothermal parameters in $z<1$, i.e.,

$$
\begin{aligned}
& F_{x}^{2}=u_{x}^{2}+v_{x}^{2}+w_{x}^{2}=u_{y}^{2}+v_{y}^{2}+w_{y}^{2}=F_{y}^{2} \\
& F_{x} \cdot F_{y}=u_{x} u_{y}+v_{x} v_{y}+w_{x} w_{y}=0 \text { for }|z|<1
\end{aligned}
$$

(c) $F\left(e^{i \theta}\right)$ is a homeomorphism of $|z|=1$ with $\Gamma$.

A solution to Plateau's problem for $\Gamma$ is a generalized minimal surface spanning $\Gamma$, and a solution may be normalized by specifying that three fixed points on $|z|=1$ correspond to three fixed points on $\Gamma$. We shall consider the solutions to be normalized, and we note that there may be more than one normalized surface spanning a given curve $\Gamma$.

Consider the analytic functions of which $u, v, w$ are the real parts:

$$
\lambda(z)=u(z)+i u^{*}(z) \quad \mu(z)=v(z)+i v^{*}(z) \quad \nu(z)=w(z)+i w^{*}(z) .
$$

Then the condition (b) is equivalent to

$$
\lambda^{\prime 2}(z)+\mu^{\prime 2}(z)+\nu^{\prime 2}(z)=0 \quad|z|<1 .
$$


This paper will deal with the differentiability of $\lambda, \mu, \nu$ at the boundary $|z|=1$, under given smoothness conditions on the curve $\Gamma$.

It was noted by Weierstrass that if the boundary $\Gamma$ of a minimal surface $S$ contains a straight line segment $\alpha$, then the surface may be extended analytically as a minimal surface across $\alpha$, by use of the reflection principle. In $1951 \mathrm{H}$. Lewy [5] proved that if $\alpha$ is an analytic arc then the surface can be extended analytically across $\alpha$.

For an up-to-date account of the studies on the boundary behavior of minimal surfaces see the recent paper of J. C. C. Nitsche [7]. In that paper Nitsche proved among other results that if $\Gamma \in C^{n, \alpha}$ for $n \geqq 1$ and $0<\alpha<1$, then $F(z) \in C^{n, \alpha}$ in $|z| \leqq 1$ and the Hölder constant for the $n$th derivatives of $F(z)$ is the same for all solutions of Plateau's problem, i.e., they depend only on the geometrical properties of $\Gamma$. In this connection see also [4], where a completely different proof of the first part of Nitsche's theorem is given.

In the following we shall say that a function $f(z) \in C^{n, \omega(t)}$ for $z$ in some domain if $f^{(n)}$ exists and has modulus of continuity $\omega(t)$, i.e.,

$$
\left|f^{(n)}\left(t_{1}\right)-f^{(n)}\left(t_{2}\right)\right| \leqq \omega\left(\left|t_{1}-t_{2}\right|\right) \quad \text { for } \quad\left|t_{1}-t_{2}\right|<\sigma,
$$

where $\omega(t)$ is a nondecreasing, non-negative function for $0 \leqq t \leqq \sigma$ and $\int_{0}^{o}(\omega(t) / t) d t<\infty$. We shall assume, as we may without loss of generality, that $t=O(\omega(|t|))$ as $t \rightarrow 0$. In the following $O(\varphi(t))$ shall mean $O(\varphi(t))$ as $t \rightarrow 0$. Note that if $\omega(t)=k t^{\alpha}, 0<\alpha<1, k$ a constant, then $f(t) \in C^{n, \alpha}$. We shall denote by $s(\theta)=s\left(F\left(e^{i j}\right)\right)$ the arclength along $\Gamma$ with $s(0)=0$. Our principal results are the following.

Theorem 1. If $\Gamma \in C^{1, \alpha}, 0<\alpha \leqq 1$ then each of $\lambda, \mu, \nu$ is continuously differentiable in $|z| \leqq 1$. In addition, there exists a constant $c$ such that $\left|s^{\prime}(\theta)\right| \leqq c,-\pi \leqq \theta \leqq \pi$, where $c$ is dependent only on $\Gamma$.

Theorem 2. Suppose $\Gamma \in C^{1, \omega(t)}$ and $\lambda, \mu, \nu$ are continuously differentiable for $|z| \leqq 1$. Let $c$ be a constant such that $\max _{|\theta| \leqq \pi}\left|s^{\prime}(\theta)\right| \leqq c$ and let $\omega_{0}(t)=\omega(c t)$. Then there exist constants $K$ and $K_{1}$ depending on $c$ and on $\omega(t)$, such that $\lambda^{\prime}\left(e^{i \gamma}\right), \mu^{\prime}\left(e^{i q}\right), \nu^{\prime}\left(e^{i \vartheta}\right)$ have modulus of continuity

$$
\omega_{0}^{*}(\theta)=K\left(\int_{0}^{\theta} \frac{\omega_{0}(t)}{t} d t+\theta \int_{\theta}^{\pi} \frac{\omega_{0}(t)}{t^{2}} d t\right)
$$

and $\lambda^{\prime}(z), \mu^{\prime}(z), \nu^{\prime}(z)$ have modulus of continuity $K_{1} \omega_{0}^{*}(\pi t)$ for $|z| \leqq 1$.

Combining Theorems 1 and 2 we obtain: If $\Gamma \in C^{1, \alpha}, 0<\alpha<1$ then $\lambda, \mu, \nu \in C^{1, \alpha}$ for $|z| \leqq 1$. If $\Gamma \in C^{1,1}$ then $\lambda, \mu, \nu \in C^{1, \omega^{*}(t)}$ for $\omega^{*}(t)=K t \log 3 \pi / t$ for some constant $K$. Furthermore there exists a constant $c$ such that $\left|s^{\prime}(\theta)\right| \leqq c$ for all $|\theta| \leqq \pi$. K and $c$ depend on 
$\Gamma$ only.

THEOREM 3. Suppose that $\Gamma \in^{n, \omega(t)}, n \geqq 2$. Let $c$ be a constant such that $\left|s^{\prime}(\theta)\right| \leqq c,|\theta| \leqq \pi$, and let $\omega_{0}(t)=\omega(c t)$ (such a constant $c$ which depends only on $\Gamma$ exists by Theorem 1). Then:

(i) $\lambda^{(n)}, \mu^{(n)}, \nu^{(n)}$ have continuous extensions to $|z|=1$ and there exist constants $K$ and $K_{1}$, depending only on $\Gamma$ such that $\lambda^{(n)}\left(e^{i \theta}\right), \mu^{(n)}\left(e^{i \theta}\right)$, $\nu^{(n)}\left(e^{i \theta}\right)$ have modulus of continuity

$$
\omega_{0}^{*}(\theta)=K\left[\int_{0}^{\theta} \frac{\omega_{n}(t)}{t} d t+\theta \int_{\theta}^{\pi} \frac{\omega_{0}(t)}{t^{2}} d t\right]
$$

and $\lambda^{(n)}(z), \mu^{(n)}(z), \nu^{(n)}(z)$ have modulus of continuity $K_{1} \omega_{0}^{*}(\pi t)$ for $|z| \leqq 1$.

(ii) There exists a constant $c_{n}$ depending only on $\Gamma, n$ such that $\left|s^{(n)}(\theta)\right| \leqq c_{n}$ for $|\theta| \leqq \pi$.

Conformal mappings in the plane are special cases of minimal surfaces and in the conformal mapping case the result for $\omega(t)=K t^{\alpha}$, $0<\alpha<1$ is due to 0 . D. Kellogg. The extension of Kellogg's theorem to a modulus of continuity satisfying a Dini condition $\int_{0}^{o}(\omega(t) / t) d t<\infty$, was given by S. E. Warschawski [8] for $n=1$ (for $n>1$ see [9]).

The case $\Gamma \in C^{1, \omega(t)}$, i.e., the proof of Theorem 3 for $n=1$, does not seem to lend itself to the method we use in establishing our Theorem 1. However, Warschawski [10] has recently given a proof of this case along different lines.

We note that our results overlap to some extent with those of Nitsche [7]. They were obtained independently, although a basic device used in the proof of Theorem 1 (Lemmas 5 and 6) is the same. However, there are differences both in approach and in detail between the two proofs.

The results hold for minimal surfaces in $n$-space, in which case we have $n$ harmonic and $n$ analytic functions. Also, it will be apparent that the theorems are local in the sense that they are true for subarcs of $\Gamma$.

2. Auxiliary Results. In the following we shall need a number of lemmas.

Lemma 1. Suppose that the function $f(z)=u\left(r e^{i t}\right)+i u^{*}\left(r e^{i t}\right)$ is holomorphic in $|z|<1$ and $u\left(r e^{i t}\right)$ is continuous in $|z| \leqq 1$. Suppose also that for some integer $n \geqq 0$

$$
\left|u\left(e^{i t}\right)\right| \leqq A|t|^{n} \omega(|t|) \quad \text { for }|t| \leqq \pi
$$


where $A$ is a constant and $\omega(t)$ is nondecreasing and nonnegative.

Then there exists a constant $M$, depending only on $A$ and on $n$, such that for $r \geqq 1 / 2$,

$$
\left|f^{(n+1)}(r)\right| \leqq M \int_{1-r}^{\pi} \frac{\omega(t)}{t^{2}} d t .
$$

Proof. We begin with the Poisson Integral for $f$ :

$$
f(z)=\frac{1}{2 \pi} \int_{-\pi}^{\pi} u\left(e^{i t}\right) \frac{e^{i t}+z}{e^{i t}-z} d t+i u^{*}(0) \quad|z|<1 .
$$

Differentiating, we obtain

$$
f^{(n+1)}(z)=\frac{(n+1) !}{\pi} \int_{-\pi}^{\pi} \frac{u\left(e^{i t}\right) e^{i t}}{\left(e^{i t}-z\right)^{n+2}} d t
$$

and in particular

$$
\begin{aligned}
\left|f^{(n+1)}(r)\right| & \leqq \frac{2 A(n+1) !}{\pi} \int_{0}^{\pi} \frac{t^{n} \omega(t)}{\left[1-2 r \cos t+r^{2}\right]^{n / 2+1}} d t \\
& \leqq \frac{2 A(n+1) !}{\pi} \int_{0}^{\pi} \frac{t^{n} \omega(t)}{\left[(1-r)^{2}+4 r \frac{t^{2}}{\pi^{2}}\right]^{n / 2+1}} d t \\
& \leqq \frac{2 A(n+1) !}{\pi}\left[\int_{0}^{1-r} \frac{t^{n} \omega(t)}{(1-r)^{n+2}} d t+\int_{1-r}^{\pi} \frac{t^{n} \omega(t)}{\left[4 r \frac{t^{2}}{\pi^{2}}\right]^{n / 2+1}} d t\right] \\
& \leqq \frac{2 A(n+1) !}{\pi}\left[\frac{\omega(1-r)}{(1-r)^{n+2}} \int_{0}^{1-r} t^{n} d t+\frac{\pi^{n+2}}{2^{n / 2+1}} \int_{1-r}^{\pi} \frac{t^{n} \omega(t)}{t^{n+2}} d t\right]
\end{aligned}
$$

for $r \geqq 1 / 2$,

$$
\leqq \frac{2 A n !}{\pi} \frac{\omega(1-r)}{1-r}+\frac{A(n+1) ! \pi^{n+1}}{2^{n / 2}} \int_{1-r}^{\pi} \frac{\omega(t)}{t^{2}} d t .
$$

Now

$$
\int_{1-r}^{\pi} \frac{\omega(t)}{t^{2}} d t \geqq \omega(1-r)\left[\frac{1}{1-r}-\frac{1}{\pi}\right]>\frac{1}{2} \frac{\omega(1-r)}{1-r}
$$

so that we may choose $M$ depending only on $A$ and on $n$ such that

$$
\left|f^{(n+1)}(r)\right| \leqq M \int_{1-r}^{\pi} \frac{\omega(t)}{t^{2}} d t \quad \text { for } \quad r \geqq \frac{1}{2} .
$$

In the case $n=0, \omega(t)=t^{\alpha} 0<\alpha<1$ we have here a result of Hardy and Littlewood (see [2] p. 360-366): If the conditions on $u$ and $f$ are satisfied and if $\left|u\left(e^{i t}\right)\right| \leqq A|t|^{\alpha}, 0<\alpha \leqq 1,|t|<\pi$ then there exists a constant $M$ depending on $A$ such that for $r \geqq 1 / 2$, 


$$
\left|f^{\prime}(r)\right| \leqq \frac{M}{(1-r)^{1-\alpha}} \quad \text { if } \quad 0<\alpha<1
$$

and

$$
\left|f^{\prime}(r)\right| \leqq M \log \frac{\pi}{1-r} \quad \text { if } \quad \alpha=1 .
$$

For our study of the higher derivatives it is useful to extend Lemma 1.

Lemma 2. Suppose that $f(z)=u\left(r e^{i t}\right)+i u^{*}\left(r e^{i t}\right)$ satisfies the hypotheses of Lemma 1 and that for $n \geqq 0$

$$
u\left(e^{i t}\right)=\sum_{i=0}^{n} a_{i} t^{i}+O\left(|t|^{n} \omega(|t|)\right) \quad \text { for }|t| \leqq \pi
$$

where $\omega(t)$ is nondecreasing, nonnegative and $t=O(\omega(|t|))$. Then there exists a constant $M$ depending only on $n$, on the $\left\{a_{i}\right\}$ and on the constant in the $O\left(|t|^{n} \omega(|t|)\right)$ term such that for $r \geqq 1 / 2$,

$$
\left|f^{(n+1)}(r)\right| \leqq M \int_{1-r}^{\pi} \frac{\omega(t)}{t^{2}} d t
$$

Proof. Let

$$
\begin{aligned}
p_{k}(t) & =\operatorname{Re} \frac{\left(e^{i t}-1\right)^{k}}{i^{k}}=\operatorname{Re}\left[\frac{i^{k} t^{k}}{i^{k}}+\frac{k}{2} \frac{i^{k+1} t^{k+1}}{i^{k}}+\cdots\right] \\
& =\sum_{j=k}^{n} a_{\jmath k} t^{j}+O\left(|t|^{n+1}\right) \quad a_{k k}=1 \quad 0 \leqq k \leqq n .
\end{aligned}
$$

Then consider

$$
\sum_{k=0}^{n} x_{k} p_{k}(t)=\sum_{k=0}^{n} x_{k}\left[\sum_{j=k}^{n} a_{j_{k}} t^{j}+O\left(|t|^{n+1}\right)\right]
$$

where the real constants $x_{k}$ are chosen so that

$$
\sum_{k=0}^{n} x_{k}\left(\sum_{j=k}^{n} a_{j k} t^{j}\right)=\sum_{j=0}^{n} a_{j} t^{j} ;
$$

this may be done as these $x_{k}$ are the solutions of the equation

$$
\left(\begin{array}{cccccc}
a_{00} & 0 & 0 & \cdots & 0 \\
a_{10} & a_{11} & 0 & \cdots & . & 0 \\
\vdots & \vdots & & & \vdots \\
a_{n 0} & a_{n 1} & & \cdots & a_{n n}
\end{array}\right)\left(\begin{array}{c}
x_{0} \\
x_{1} \\
\vdots \\
x_{n}
\end{array}\right)=\left(\begin{array}{c}
a_{0} \\
a_{1} \\
\vdots \\
a_{n}
\end{array}\right) .
$$

We then set

$$
p(z)=\sum_{k=0}^{n} x_{k} \frac{(z-1)^{k}}{i^{k}} .
$$


Now let $g(z)=f(z)-p(z)$. Then $g$ is holomorphic for $|z|<1$, continuous for $|z| \leqq 1, g^{(n+1)}(z) \equiv f^{(n+1)}(z)$ and

$$
\begin{aligned}
\left|\operatorname{Re} g\left(e^{i t}\right)\right| & =\left|\operatorname{Re}\left[f\left(e^{i t}\right)-p\left(e^{i t}\right)\right]\right| \\
& =\left|u\left(e^{i t}\right)-\sum_{k=0}^{n} x_{k} p_{k}(t)\right| \\
& =O\left(|t|^{n} \omega(|t|)\right)+O\left(|t|^{n+1}\right)=O\left(|t|^{n} \omega(|t|)\right)
\end{aligned}
$$

since $t=O(\omega(|t|))$. Thus by Lemma 1

$$
\left|f^{(n+1)}(r)\right|=\left|g^{(n+1)}(r)\right| \leqq M \int_{1-r}^{\pi} \frac{\omega(t)}{t^{2}} d t
$$

where the constant $M$ depends only on the constant in the $O$-term in (4). Now note that the $\left\{a_{j k}\right\}$ are totally independent of the function $u$, so the $\left\{x_{i}\right\}$ are dependent only on the $\left\{a_{i}\right\}$. The $\left\{x_{i}\right\}$ affect the constant in the $O\left(t^{n} \omega(|t|)\right)$ term in (4) via (3) so that the constant in (4) depends only on the $\left\{a_{i}\right\}$ and the $O\left(|t|^{n} \omega(|t|)\right)$ term in (2). Thus the value of $M$ depends only on these constants.

COROLLARY. If the conditions of Lemma 2 are satisfied and if $\int_{0}^{\pi}(\omega(t) / t) d t<\infty$, then there exists a constant $A$ dependent only on the $\left\{a_{i}\right\}, \omega(t), n$, and the constant in the $O$ term in (2), such that for $r \geqq 1 / 2$

$$
\left|f^{(n)}(r)\right| \leqq A
$$

Proof. Let $A_{1}$ be the constant in the $O$ term in (4). Then as in the proof of Lemma 1 ,

$$
\begin{aligned}
\left|f^{(n)}(r)-p^{(n)}(r)\right| & \leqq \frac{n ! A_{1}}{\pi} \int_{0}^{\pi} \frac{t^{n} \omega(t)}{\left(\frac{4 r t^{2}}{\pi^{2}}\right)^{(n+1) / 2}} d t \\
& \leqq \frac{n ! A_{1} \pi^{n}}{2^{(n+1) / 2}} \int_{0}^{\pi} \frac{\omega(t)}{t} d \theta=A_{2}
\end{aligned}
$$

so that

$$
\left|f^{(n)}(r)\right| \leqq A_{2}+\left|p^{(n)}(r)\right|
$$

But $p^{(n)}(r)=n ! x_{n}$ and $x_{n}$ depends on the $\left\{a_{i}\right\}$ so

$$
\left|f^{(n)}(r)\right| \leqq A_{2}+n ! x_{n}=A \text {. }
$$

Lemma 3. Suppose $f(z)$ is holomorphic in $|z|<1$ and $f^{\prime}(z)$ satisfies the condition

$$
\left|f^{\prime}\left(r e^{i \vartheta}\right)\right| \leqq M \int_{1-r}^{\pi} \frac{\omega(t)}{t^{2}} d t
$$


for all $|\theta| \leqq \pi$ and for all $0<r<1$. Here $M$ is a constant and $\omega(t)$ is nondecreasing, nonnegative, bounded for $0 \leqq t \leqq \pi$, and $\int_{0}^{\pi}(\omega(t) / t) d t<\infty$. Then,

(i ) $\lim _{r \rightarrow 1} f\left(r e^{i \theta}\right)=f\left(e^{i \theta}\right)$ exists and is finite for $|\theta| \leqq \pi$ and $f\left(e^{i \theta}\right)$ has the modulus of continuity

$$
\omega^{*}(\theta)=3 M\left[\int_{0}^{\theta} \frac{\omega(t)}{t} d t+\theta \int_{\theta}^{\pi} \frac{\omega(t)}{t^{2}} d t\right] .
$$

(ii) $f(z)$ is continuous in $|z| \leqq 1$ and has modulus of continuity $A \omega^{*}(\pi t)$ where $A$ is a constant depending only on the function $\omega^{*}(t)$. That is, for $\left|z_{1}\right|,\left|z_{2}\right| \leqq 1$,

$$
\left|f\left(z_{2}\right)-f\left(z_{1}\right)\right| \leqq A \omega^{*}\left(\pi\left|z_{2}-z_{1}\right|\right) .
$$

Here we define $\omega^{*}(t)=\omega^{*}(\pi)$ for $t \geqq \pi$.

For the proof of part (i) see [10], Lemma 4; the proof of part (ii) is patterned after that of the more special theorem in [2], page 363.

In the case $\omega(t)=t^{\alpha}, 0<\alpha<1$ this is another result of Hardy and Littlewood ([2] Pages 360-366):

If $f$ is as in Lemma 3 and if $\left|f^{\prime}\left(r e^{i j}\right)\right| \leqq M /(1-r)^{1-\alpha}$ for all $|\theta| \leqq \pi$ then $f\left(e^{i \theta}\right) \in \operatorname{Lip}\left(\alpha^{\prime}\right)$ for $|\theta| \leqq \pi$. If $\omega(t)=t$ then $\left|f^{\prime}\left(r e^{i \theta}\right)\right| \leqq$ $M \log (\pi /(1-r))$ and the conclusion is that $f\left(e^{i \theta}\right)$ has modulus of continuity $\omega^{*}(t)=3 M t \log (3 \pi / t)$.

We note that a result analogous to Lemma 3 can be obtained if (5) is satisfied for a subarc $\theta_{1} \leqq \theta \leqq \theta_{2}$ of $|z|=1$ for $0<r<1$. Then $f\left(e^{i \theta}\right)$ has modulus of continuity $\omega^{*}(t)$ on this arc and $f(z)$ has modulus of continuity $A \omega^{*}(\pi t)$ in the sector $\theta_{1} \leqq \theta \leqq \theta_{2}, 0 \leqq r \leqq 1, A$ depending on $\omega^{*}$. Thus it will be evident that our theorems will hold for subares of $\Gamma$.

The first link between the geometry of $\Gamma$ and the function $F$ is given by the following Lemma, (see [8] pp. 615-17 and [6] p. 238).

Lemma 4. Suppose $\Gamma$ is a closed Jordan curve in $R^{3}$ and $F(z)$ is a solution to Plateau's problem for $\Gamma$. For two points $p_{1}, p_{2} \in \Gamma$, let $\Delta s\left(p_{1} p_{2}\right)$ denote the length of the shorter arc between $p_{1}$ and $p_{2}$. Suppose there exist constants $c>1$ and $\delta>0$ such that $\Delta s\left(p_{1} p_{2}\right) / \overline{p_{1} p_{2}}<c$ for $\Delta s\left(p_{1} p_{2}\right)<\delta$. Then there exist constants $K>0, \delta_{1}>0$, depending on $\Gamma$ only, such that for $\left|\theta-\theta_{0}\right|<\delta_{1}$

$$
\left|F\left(e^{i \theta}\right)-F\left(e^{i \theta_{0}}\right)\right| \leqq\left|s(\theta)-s\left(\theta_{0}\right)\right| \leqq K\left|\theta-\theta_{0}\right|^{\beta}
$$

where $s(\theta)$ for $|\theta| \leqq \pi$ is arclength along $\Gamma$ and where $\beta=2 /(1+c)^{2}$ so that $0<\beta<1 / 2$. 

of $F$.

Proof. Let $D[F]=1 / 2 \iint_{|z|<1}\left(F_{x}^{2}+F_{y}^{2}\right) d x d y$, the Dirichlet integral

If there exists a constant $B$ such that for each solution $F$ to Plateau's problem, $D[F] \leqq B$, then Lemma 3.2 of [1] implies that the family of solutions is equicontinuous. Since $x$ and $y$ are isothermal coordinates $D[F]=A[F]$, the area of the minimal surface, and by the isoperimetric inequality for minimal surfaces, $A[F] \leqq L^{2} / 4 \pi$ where $L$ is the length of $\Gamma$. Thus $D[F] \leqq L^{2} / 4 \pi=B$ for all minimal surfaces spanning $\Gamma$ which satisfy the three point condition and, as the modulus of continuity of the vectors $\left\{F\left(e^{i \theta}\right)\right\}$ depends only on $B$, it depends only on $\Gamma$. Thus the family of arclength functions $\{s(\theta)\}$ associated with the minimal surfaces has a uniform modulus of continuity which depends only on $\Gamma$.

Let $D$ be the diameter of $\Gamma$ and let $\delta^{\prime}>0$ be such that $\left|\theta-\theta^{\prime}\right|<\delta^{\prime}$ implies $\left|s(\theta)-s\left(\theta^{\prime}\right)\right|<\min (\delta, D / 2)$ for all minimal surface spanning $\Gamma$.

Let $k_{\rho}=\left\{z:\left|z-e^{i \theta_{0}}\right|=\rho,|z|<1\right\}$ where $\rho<\min \left(\delta^{\prime} / 4,1\right)$ and let $e^{i \theta_{2}}$ and $e^{i \theta_{1}}$ be the endpoints of $k_{\rho}$ which are on $|z|=1$. Then $\left|\theta_{2}-\theta_{1}\right|<\delta^{\prime}$ so $\left|s\left(\theta_{2}\right)-s\left(\theta_{1}\right)\right|<\min (\delta, D / 2)$. Thus $F\left(e^{i \theta_{0}}\right)$ must be on the shorter arc between $F\left(e^{i \theta_{2}}\right)$ and $F\left(e^{i \theta_{1}}\right)$. This is true for all solutions to the Plateau problem for $\Gamma$.

Now let $l_{\rho}=$ length of $F\left(k_{\rho}\right)$. Then, for $z_{0}=e^{i 0_{0}}$

$$
l_{\rho}=\int_{k_{\rho}}\left|F_{\varphi}\left(z_{0}+\rho e^{i \varphi}\right)\right| d \varphi
$$

and by Schwarz's inequality

so that

$$
l_{\rho}^{2} \leqq \pi \int_{k_{\rho}}\left|F_{\varphi}\left(z_{0}+\rho e^{i \varphi}\right)\right|^{2} d \varphi
$$

$$
\frac{l_{\rho}^{2}}{\rho} \leqq \pi \int_{k_{\rho}} \frac{1}{\rho^{2}}\left|F_{\varphi}\left(z_{0}+\rho e^{i \varphi}\right)\right|^{2} \rho d \varphi .
$$

Since $F$ is a minimal surface $1 / \rho^{2} \cdot F_{\varphi}^{2}=F_{\rho}^{2}$ so that $1 / \rho^{2} \cdot F_{\varphi}^{2}=1 / 2\left(F_{\rho}^{2}+\right.$ $\left.1 / \rho^{2} \cdot F_{\varphi}^{2}\right)$ and thus

$$
\int_{0}^{r} \frac{l_{\rho}^{2}}{\rho} d \rho \leqq \frac{\pi}{2} \int_{0}^{r} \int_{k_{\rho}}\left(F_{\rho}^{2}+\frac{1}{\rho^{2}} F_{\varphi}^{2}\right) \rho d \varphi d \rho .
$$

Letting $\Delta_{r}=F\left(\left\{z:\left|z-e^{i \theta_{0}}\right| \leqq r,|z|<1\right\}\right)$ and $A(r)=$ area of $\Delta_{r}$, we have

$$
\mathscr{F}(r):=\int_{0}^{r} \frac{l_{\rho}^{2}}{\rho} d \rho \leqq \pi A(r)
$$

Let $L$ denote the length of the boundary of $\Delta_{r}$. By the isoperimetric inequality $A(r) \leqq L^{2} / 4 \pi$. By our first remarks letting $p_{1}=F\left(e^{i \theta_{1}}\right)$ 
and $p_{2}=F\left(e^{i \theta_{2}}\right)$, we have

so that

$$
L=l_{r}+\Delta s\left(p_{1} p_{2}\right) \leqq l_{r}+c \overline{p_{1} p_{2}} \leqq(1+c) l_{r}
$$

$$
\mathscr{F}(r) \leqq \frac{\pi L^{2}}{4 \pi}=\frac{L^{2}}{4} \leqq \frac{l_{r}^{2}(1+c)^{2}}{4} .
$$

Now $\mathscr{F}^{\prime}(r)=l_{r}^{2} / r$ a.e., so $r \mathscr{F}^{\prime}(r)=l_{r}^{2}$ and $\mathscr{F}(r) \leqq(1+c)^{2} / 4 \cdot r \mathscr{F}^{\prime}(r)$. Then for $\rho<\rho_{0}=\min \left(\delta^{\prime} / 4,1\right)$

$$
\frac{4}{(1+c)^{2}} \int_{\rho}^{\rho_{0}} \frac{d r}{r} \leqq \int_{\rho}^{\rho_{0}} \frac{\mathscr{F}^{\prime}(r)}{\mathscr{F}^{\prime}(r)} d r
$$

so that

$$
\left(\frac{\rho_{0}}{\rho}\right)^{4 /(1+c)^{2}} \leqq \frac{\mathscr{F}\left(\rho_{0}\right)}{\mathscr{F}(\rho)}
$$

Choose $M$ so that $\mathscr{F}(\rho) /\left(\rho^{4 /(1+c)^{2}}\right) \leqq \mathscr{F}\left(\rho_{0}\right) /\left(\rho_{0}^{\left.4 /(1+c)^{2}\right)}\right)=M-1 . \quad M$ depends only on $\Gamma$ since $\mathscr{F}\left(\rho_{0}\right) \leqq \pi A\left(\rho_{0}\right) \leqq \pi A[F] \leqq L^{2} / 4 \pi=B$ and $\rho_{0}$ depends only on $\delta^{\prime}$. Then $\mathscr{F}(\rho)<M \rho^{4 /(1+c)^{2}}$ so that

$$
\int_{\rho / 2}^{\rho} \frac{l_{r}^{2}}{r} d r \leqq \int_{0}^{\rho} \frac{l_{r}^{2}}{r} d r<M \rho^{4 /(1+c)^{2}} \text {. }
$$

Now there exists a $\rho_{1}$ with $\rho / 2 \leqq \rho_{1} \leqq \rho$ such that

$$
l_{\rho_{1}}^{2} \int_{\rho / 2}^{\rho} \frac{d r}{r}<M \rho^{4 /(1+c)^{2}}
$$

so that

and thus

$$
l_{\rho_{1}}^{2} \log 2<M \rho^{4 /(1+c)^{2}}
$$

$$
l_{\rho_{1}}<\sqrt{\frac{M}{\log 2}} \rho^{2 /(1+c)^{2}}=\sqrt{\frac{M}{\log 2}} \rho^{\beta} .
$$

Thus if $\left|e^{i \theta}-e^{i \theta_{0}}\right|=\rho / 2$ and if $p_{1}=F\left(e^{i \theta_{1}}\right)$ and $p_{2}=F\left(e^{i \theta_{2}}\right)$ are the endpoints of $k_{\rho_{1}}$

$$
\begin{aligned}
\left|F\left(e^{i \theta}\right)-F\left(e^{i \theta_{0}}\right)\right| & \leqq\left|s(\theta)-s\left(\theta_{0}\right)\right| \leqq c \overline{p_{1} p_{2}} \\
& \leqq c \sqrt{\frac{M}{\log 2}} \rho^{\beta} \leqq c \sqrt{\frac{M}{\log 2}} 2^{\beta}\left|\theta-\theta_{0}\right|^{\beta} .
\end{aligned}
$$

Letting $K=e \sqrt{\frac{M}{\log 2}} 2^{\beta}$ we have

$$
\left|F\left(e^{i \theta}\right)-F\left(e^{i \theta_{0}}\right)\right| \leqq\left|s(\theta)-s\left(\theta_{0}\right)\right| \leqq K\left|\theta-\theta_{0}\right|^{\beta} .
$$

This is true for $\left|\theta-\theta_{0}\right|<1 / 3 \min \left(\delta^{\prime} / 4,1\right)=\delta_{1}$, for we may then choose $\rho$ so that $\rho=2\left|e^{i \theta}-e^{i \theta_{0}}\right|<2\left|\theta-\theta_{0}\right|<\rho_{0}=\min \left(\delta^{\prime} / 4,1\right)$.

Since $s(\theta)$ is bounded we may find a constant $K_{1}$ such that $\left|s(\theta)-s\left(\theta_{0}\right)\right| \leqq K_{1}\left|\theta-\theta_{0}\right|^{\beta}$ for all $\theta, \theta_{0} \in[-\pi, \pi]$. It is in this form that we shall use Lemma 4 . ( $K_{1}$ clearly depends on $\Gamma$ only.) 
For the hypothesis of Lemma 4 to hold, it is sufficient that $\Gamma$ be continuously differentiable with respect to arclength. Then $c$ may be taken as close to 1 as we like, so that $\beta$ is as close to $1 / 2$ as we like. The constant $K_{1}$ will depend on $c$, but will be uniform for all solutions to the Plateau problem for $\Gamma$.

3. The first derivative. We first prove Theorem 1. From Lemma 4 we know that $F\left(e^{i \theta}\right) \in \operatorname{Lip}(\beta)$ for any $0<\beta<1 / 2$. Our first step is to improve the Hölder exponent by a "bootstrap" technique involving the Hardy-Littlewood forms of Lemmas 1 and 3.

Lemma 5. Suppose $\Gamma$ is a smooth closed Jordan curve and $F(z)$ is a minimal surface spanning $\Gamma$. Suppose $F(1)=(0,0,0)$ and the tangent to $\Gamma$ at $F(1)$ is along the positive u axis. Let $\mathscr{F}(s)=(U(s)$, $V(s), W(s))$ be the parametrization of $\Gamma$ with respect to arclength $s$. Let $s(\theta)=s\left(F\left(e^{i \theta}\right)\right)$ and $s(0)=0$, so that $\mathscr{F}(0)=F(1)=(0,0,0)$ and $\mathscr{F}^{\prime}(0)$ is along the positive $u$ axis.

Suppose that $\mathscr{F}(s) \in C^{1, \alpha}$ for some $0<\alpha \leqq 1$ and that $F\left(e^{i \theta}\right) \in \operatorname{Lip}(\beta)$ for some $\beta>0$, with Hölder constant $K_{\beta}$.

Then there exists a constant $K$, depending only on $\Gamma, K_{\beta}$, and $\beta$, such that for $|\theta| \leqq \pi$

$$
\left|v\left(e^{i 0}\right)\right| \leqq K|\theta|^{\beta(1+\alpha)} \quad\left|w\left(e^{i \theta}\right)\right| \leqq K|\theta|^{3(1+\alpha)} .
$$

Proof. Since $V(s) \in C^{1, \alpha}$ and $V_{s}(0)=0$ we have, for some constant $K_{0}$

$$
\left|V_{s}(s)\right| \leqq K_{0}|s|^{\alpha}
$$

Since $V(0)=0$ we integrate to obtain

$$
|V(s)| \leqq \frac{K_{0}}{1+\alpha}|s|^{1+\alpha}
$$

$F(\theta) \in \operatorname{Lip}(\beta)$ implies that $s(\theta) \in \operatorname{Lip}(\beta)$ so that there exists $K_{\beta}^{\prime}$ (depending on $K_{\beta}$ and $\Gamma$ ) such that

$$
|s(\theta)| \leqq K_{\beta}^{\prime}|\theta|^{\beta}
$$

combining (6) and (7) one obtains

$$
\left|v\left(e^{i \theta}\right)\right|=|V(s(\theta))| \leqq \frac{K_{0}}{1+\alpha}\left(K_{\beta}^{\prime}\right)^{1+\alpha}|\theta|^{\beta^{(1+\alpha)}}=K|\theta|^{\beta(1+\alpha)} .
$$

The proof for $w\left(e^{i \theta}\right)$ is analogous.

We now apply Lemma 5 to raise the Hölder exponent for $F\left(e^{i \theta}\right)$.

Lemma 6. Suppose $\Gamma$ is a closed Jordan curve and $F(z)$ is a minimal surface spanning $\Gamma$. Suppose $\Gamma \in C^{1, \alpha}$ for $0<\alpha \leqq 1$ and. 
that $F\left(e^{i \theta}\right) \in \operatorname{Lip}(\beta)$ with Hölder constant $K_{\beta}$, where $\beta(1+\alpha)<1$. Then $\left(F\left(e^{i \theta}\right) \in \operatorname{Lip}(\beta(1+\alpha))\right.$ with the Hölder constant depending only on $K_{\beta}$ and $\Gamma$.

Proof. First assume that $\Gamma, F$ are in the position of Lemma 5 . Then $\left|v\left(e^{i \theta}\right)\right| \leqq K|\theta|^{\beta(1+\alpha)}$ and $\left|w\left(e^{i \theta}\right)\right| \leqq K|\theta|^{\beta(1+\alpha)}$.

Consider now $\mu(z)=v(z)+i v^{*}(z)$ and $\nu(z)=w(z)+i w^{*}(z)$. Then by Lemma $1(n=0)$, there exists a constant $M$ depending only on $K$

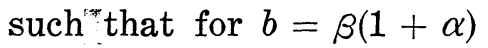

$$
\left|\mu^{\prime}(r)\right| \leqq \frac{M}{(1-r)^{1-b}} \quad \text { and } \quad\left|\nu^{\prime}(r)\right| \leqq \frac{M}{(1-r)^{1-b}} .
$$

Letting $\lambda(z)=u(z)+i u^{*}(z)$ and applying (1) we have

and hence

$$
\left|\lambda^{\prime}(z)\right|^{2} \leqq\left|\mu^{\prime}(z)\right|^{2}+\left|\nu^{\prime}(z)\right|^{2}
$$

$$
\left|\lambda^{\prime}(r)\right| \leqq \frac{\sqrt{2} M}{(1-r)^{1-b}}
$$

We would now like to apply Lemma 3 to conclude that $\lambda, \mu, \nu \in$ $\operatorname{Lip}(\beta(1+\alpha))$.

For any $F\left(e^{i \theta}\right)$ on $\Gamma$, let $\left(u^{\theta}, v^{\theta}, w^{\theta}\right)$ be a new coordinate system centered at $F\left(e^{i \theta}\right)$ and such that the $u^{\theta}$ axis is tangent to $\Gamma$ at $F\left(e^{i \theta}\right)$. Then $\left(u^{\theta}(z), v^{\theta}(z), w^{\theta}(z)\right)=F^{\theta}(z)$ is a minimal surface and by a rotation of the unit circle we may assume that $F^{\theta}(1)=F\left(e^{i 0}\right)$. It is clear that $F^{\theta}\left(e^{i t}\right) \in \operatorname{Lip}(\beta)$ with the same Hölder constant as $F\left(e^{i t}\right)$. Thus $\Gamma, F^{0}$ are as in Lemma 5, so that we may use the preceding argument to see that

$$
\left|\left(\mu^{\theta}\right)^{\prime}(r)\right| \leqq \frac{M}{(1-r)^{1-b}} \quad \text { and } \quad\left|\left(\nu^{g}\right)^{\prime}(r)\right| \leqq \frac{M}{(1-r)^{1-b}}
$$

where $\mu^{\theta}(z), \nu^{9}(z), \lambda^{\theta}(z)$ are the analytic functions with real parts $v^{\theta}(z), w^{\theta}(z)$ and $u^{\theta}(z)$, respectively and $\mu^{\theta}(1)=\nu^{\theta}(1)=\lambda^{\theta}(1)=0$ so that $\left|\left(\lambda^{\theta}\right)^{\prime}(r)\right| \leqq \sqrt{2} M /(1-r)^{1-b}$.

$M$ is dependent only on $\Gamma, \beta$ and $K_{\beta}$. If $\left(a_{i j}\right), 1 \leqq i, j \leqq 3$, is the orthogonal matrix of the coordinate transformation, we have

$$
\left\{\begin{array}{l}
\lambda\left(r e^{i \theta}\right)=a_{11}(\theta) \lambda^{\theta}(r)+a_{12}(\theta) \mu^{\theta}(r)+a_{13}(\theta) \nu^{\theta}(r)+\lambda\left(e^{i \theta}\right) \\
\mu\left(r e^{i \theta}\right)=a_{21}(\theta) \lambda^{\theta}(r)+a_{22}(\theta) \mu^{\theta}(r)+a_{23}(\theta) \nu^{\theta}(r)+\mu\left(e^{i \theta}\right) \\
\nu\left(r e^{i \theta}\right)=a_{31}(\theta) \lambda^{\theta}(r)+a_{32}(\theta) \mu^{\theta}(r)+a_{33}(\theta) \nu^{\theta}(r)+\nu\left(e^{i \theta}\right)
\end{array}\right.
$$

and therefore by the inequality of Schwarz and the orthogonality of the matrix $\left(a_{i j}\right)$

$$
\left|\lambda^{\prime}\left(r e^{i \vartheta}\right)\right| \leqq \frac{2 M}{(1-r)^{1-b}} \quad \text { for }|\theta| \leqq 2 \pi
$$


and by Lemma $3, \lambda \in \operatorname{Lip}(b)$. The same holds for $\mu$ and $\nu$, and the Hölder constant is as claimed.

LEMma 7. With $\Gamma, F$ defined as in Lemma 5, there exists an $\varepsilon>0$ such that $v\left(e^{i \theta}\right)=O\left(\theta^{1+\varepsilon}\right), w\left(e^{i \theta}\right)=O\left(\theta^{1+\varepsilon}\right)$ where the constant in $O$ depends only on $\Gamma$.

Proof. Choose $0<\beta<1 / 2$ such that for all integers $n,(1+\alpha)^{n} \neq$ $1 / \beta$. Then there exists an integer $n$ such that $(1+\alpha)^{n} \beta=1+\varepsilon>1$ but $(1+\alpha)^{n-1} \beta<1$. Apply Lemma $6 n-1$ times to obtain $F\left(e^{i \theta}\right) \epsilon$ $\operatorname{Lip}\left(\beta(1+\alpha)^{n-1}\right)$ and then apply Lemma 5 to see that there exists $K$ constant such that $|v(\theta)| \leqq K|\theta|^{1+\varepsilon}$ and $|w(\theta)| \leqq K|\theta|^{1+\varepsilon}$.

Proof of Theorem 1. First suppose $\Gamma, F$ are as in Lemma 5. Then we claim $\lim _{r \rightarrow 1} \mu^{\prime}(r)=\mu^{\prime}(1), \lim _{r \rightarrow 1} \nu^{\prime}(r)=\nu^{\prime}(1), \lim _{r \rightarrow 1} \lambda^{\prime}(r)=\lambda^{\prime}(1)$ all exist and are finite. By Lemma $7 v(\theta)=O\left(\theta^{1+\varepsilon}\right)$, hence by Lemma 1 $\left|\mu^{\prime \prime}(r)\right| \leqq M /(1-r)^{1-\varepsilon}$, for $r \leqq 1 / 2$. Then for $1 / 2 \leqq r_{1}<r_{2}<1$

$$
\begin{aligned}
\left|\mu^{\prime}\left(r_{2}\right)-\mu^{\prime}\left(r_{1}\right)\right| & =\left|\int_{r 1}^{r 2} \mu^{\prime \prime}(r) d r\right| \leqq \int_{r 1}^{r 2} \frac{M}{(1-r)^{1-\varepsilon}} d r \\
& \leqq \frac{M}{\varepsilon}\left|r_{2}-r_{1}\right|^{\varepsilon}
\end{aligned}
$$

so that $\lim _{r \rightarrow 1} \mu^{\prime}(r)=\mu^{\prime}(1)$ exists and is finite. Likewise $\lim _{r \rightarrow 1} \nu^{\prime}(r)=$ $\nu^{\prime}(1)$ exists and is finite.

Since $\lambda^{\prime 2}(r)=-\left(\mu^{\prime 2}(r)+\nu^{\prime 2}(r)\right)$, we see $\lim _{r \rightarrow 1} \lambda^{\prime}(r)=\lambda^{\prime}(1)$ exists and is finite.

From (8) it is clear that each of $\lambda^{\prime}\left(r e^{i \theta}\right), \mu^{\prime}\left(r e^{i \theta}\right), \nu^{\prime}\left(r e^{i \theta}\right)$ have radial limits for all $|\theta| \leqq \pi$ and the convergence is uniform for all $\theta$. Thus defining $\lambda^{\prime}\left(e^{i \theta}\right)=\lim _{r \rightarrow 1} \lambda^{\prime}\left(r e^{i \theta}\right)$, the function $\lambda^{\prime}\left(e^{i \theta}\right)$ is continuous. This, together with the uniform convergences of $\lambda^{\prime}\left(r e^{i \theta}\right)$ to $\lambda^{\prime}\left(e^{i \theta}\right)$ implies that $\lambda^{\prime}(z)$ is continuous for $|z| \leqq 1$. From this it follows that $\lambda(z)$ is differentiable at each $e^{i \theta}$, ie.

$$
\lim _{z \rightarrow e^{i \theta}} \frac{\lambda(z)-\lambda\left(e^{i \theta}\right)}{z-e^{i \theta}}=\lambda^{\prime}\left(e^{i \theta}\right) .
$$

The same facts are true for $\mu^{\prime}(z)$ and $\nu^{\prime}(z)$.

Finally, recall that if $\Gamma, F$ are as in Lemma 5 then there exist $\varepsilon>0$ and $K>0$ such that $\left|v\left(e^{i \theta}\right)\right| \leqq K|\theta|^{1+\varepsilon}$ and $\left|w\left(e^{i \theta}\right)\right| \leqq K|\theta|^{1+\varepsilon}$, where $K$ depends only on $\Gamma$.

Thus, by the corollary to Lemma 2 there exists a constant $K_{1}$ such that $\left|\mu^{\prime}(1)\right| \leqq K_{1}$ and $\left|\nu^{\prime}(1)\right| \leqq K_{1}$; hence $\left|\lambda^{\prime}(1)\right| \leqq \sqrt{2} K_{1}$. By the equations (8) one sees that $\left|\lambda^{\prime}\left(e^{i \theta}\right)\right|,\left|\mu^{\prime}\left(e^{i \theta}\right)\right|,\left|\nu^{\prime}\left(e^{i \theta}\right)\right|$ are bounded by $2 K_{1}$ for all $\theta$. Thus $\left|s^{\prime}(\theta)\right| \leqq 2 \sqrt{3} K_{1}=c$ for $|\theta| \leqq \pi$, and $c$ is 
the same for any solution to Plateau's problem for $\Gamma$.

We now prove a lemma preparatory to the proof of Theorem 2 .

Lemma 8. Suppose $\Gamma, F$ are positioned as in Lemma 5. Suppose also that $\lambda^{\prime}, \mu^{\prime}, \nu^{\prime}$ are continuous in $|z| \leqq 1$ and $\Gamma \in C^{1, \omega(t)}$. Let $\left|s^{\prime}(\theta)\right| \leqq c,|\theta| \leqq \pi$, and let $\omega_{0}(\theta)=\omega(c \theta)$. Then

$$
\left|v\left(e^{i \theta}\right)\right| \leqq K\left|\theta \omega_{0}(|\theta|)\right|,\left|w\left(e^{i \theta}\right)\right| \leqq K\left|\theta \omega_{0}(|\theta|)\right|,\left|u^{*}\left(e^{i \theta}\right)\right| \leqq K\left|\theta \omega_{0}(|\theta|)\right|
$$

for $|\theta| \leqq \pi$, where the constant $K$ depends only on $c$ and $\Gamma$.

Proof. By the argument of Lemma 5 we have $|V(s)| \leqq|s| \omega(s)$ and since $|s(\theta)| \leqq c|\theta|,\left|v\left(e^{i \theta}\right)\right| \leqq c|\theta| \omega_{0}(|\theta|)$; likewise $\left|w\left(e^{i \theta}\right)\right| \leqq c|\theta| \omega_{0}(|\theta|)$.

By Lemma 4, $U_{s}(s(\theta))$ is uniformly continuous for $|\theta| \leqq \pi$ and $U_{s}(s(0))=1$. Therefore there exists a $\delta>0$ (depending only on $\Gamma$ ) such that $|\theta|<\delta$ implies $U_{s}(s(\theta))>1 / 2$. Now $d s(\theta) / d \theta \neq 0$ for almost every $\theta$ and $U_{s} s_{\theta}=u_{\theta}$ and $V_{s} s_{\theta}=v_{0}$ so that

$$
\frac{v_{\theta}\left(e^{i \theta}\right)}{u_{\theta}\left(e^{i \theta}\right)}=\frac{V_{s}(s(\theta)) s_{\theta}(\theta)}{U_{s}(s(\theta)) s_{\theta}(\theta)}=\frac{V_{s}(s(\theta))}{U_{s}(s(\theta))} \quad \text { a.e. }|\theta|<\delta .
$$

But

$$
\left|\frac{V_{s}(s)}{U_{s}(s)}\right| \leqq 2 \omega(|s|) \leqq 2 \omega_{0}(|\theta|)
$$

so that

$$
\left|\frac{v_{\theta}\left(e^{i \theta}\right)}{u_{\theta}\left(e^{i \theta}\right)}\right| \leqq 2 \omega_{0}(|\theta|) \quad \text { a.e. }|\theta|<\delta ;
$$

likewise

$$
\left|\frac{w_{\theta}\left(e^{i \theta}\right)}{u_{\theta}\left(e^{i \theta}\right)}\right| \leqq 2 \omega_{0}(|\theta|) \quad \text { a.e. }|\theta|<\delta .
$$

In polar coordinates the minimal surface condition implies that $u_{r} u_{\theta}+v_{r} v_{\theta}+w_{r} w_{\theta}=0$ and therefore

$$
-u_{\theta}^{*}=-u_{r}=v_{r} \frac{v_{\theta}}{u_{\theta}}+w_{r} \frac{w_{\theta}}{u_{\theta}}
$$

but $\left|v_{r}\left(e^{i \theta}\right)\right|$ and $\left|w_{r}\left(e^{i \theta}\right)\right|$ are both bounded by $c$ for all $\theta$ so that $\left|u_{\theta}^{*}\left(e^{i \theta}\right)\right| \leqq 4 c \omega_{0}(|\theta|)$ a.e. $|\theta|<\delta$. Taking $u^{*}\left(e^{i \theta}\right)=0$ we may integrate to obtain

$$
\left|u^{*}\left(e^{i \theta}\right)\right| \leqq 4 c|\theta| \omega_{0}(|\theta|) \quad|\theta|<\delta .
$$

Since $\delta$ was dependent only on $\Gamma$ it is clear that $K$ may be chosen to complete the proof of the lemma.

Proof of Theorem 2. Suppose first that $\Gamma, F$ are as in Lemma 5. Then the conclusion of Lemma 8 holds. Applying Lemma 1 to $-i \lambda(z)$, 
for instance, we obtain

$$
\left|\lambda^{\prime \prime}(r)\right| \leqq M \int_{1-r}^{\pi} \frac{\omega_{0}(t)}{t^{2}} d t \quad \text { for } \quad r \geqq \frac{1}{2}
$$

and analogous inequalities for $\left|\mu^{\prime \prime}(r)\right|$ and $\left|\nu^{\prime \prime}(r)\right|$. Since $M$ depends only on $\Gamma$ we see by applying the transformation (8) that

$$
\left|\lambda^{\prime \prime}\left(r e^{i \theta}\right)\right| \leqq \sqrt{3} M \int_{1-r}^{\pi} \frac{\omega_{0}(t)}{t^{2}} d t \quad|\theta| \leqq \pi .
$$

Analogous inequalities hold for $\left|\mu^{\prime \prime}\left(r e^{i \theta}\right)\right|$ and $\left|\nu^{\prime \prime}\left(r e^{i 0}\right)\right|$. The conclusion of Theorem 2 then follows from Lemma 3.

4. The higher derivatives. In proving Theorem 3 for a given $n \geqq 2$, the result for $n-1$ is assumed, so that $\Gamma \in C^{n, \omega(t)}$ implies $\Gamma \in C^{n-1,1}$ and thus $s^{(n-1)}(\theta)$ has modulus of continuity $k t \log 3 \pi / t$.

We shall make extensive use of the following fact: If $f(x) \in C^{n, \omega(t)}$ for $|x| \leqq \delta$, then

$$
f(x)=\sum_{i=0}^{n} f^{(i)}(0) \frac{x^{i}}{i !}+O\left(\left|x^{n}\right| \omega(|x|)\right) .
$$

We now prove a lemma analogous to Lemma 8.

Lemma 9. Suppose $\Gamma \in C^{n, \omega(t)}, n \geqq 2$, and that $\Gamma, F$ are positioned as in Lemma 5. Suppose $c \geqq\left|s^{\prime}(\theta)\right|$ for $|\theta| \leqq \pi$ and that $\omega_{0}(\theta)=\omega(c \theta)$. Such $a c$ exists and is dependent only on $\Gamma$ by Theorem 1. Then there exist constants $\left\{b_{i}\right\},\left\{c_{i}\right\},\left\{a_{i}\right\} 2 \leqq i \leqq n$ such that

$$
\left\{\begin{array}{l}
v\left(e^{i \theta}\right)=\sum_{i=2}^{n} b_{i} \theta^{i}+O\left(|\theta|^{n} \omega_{1}(|\theta|)\right) \\
w\left(e^{i \theta}\right)=\sum_{i=2}^{n} c_{i} \theta^{i}+O\left(|\theta|^{n} \omega_{1}(|\theta|)\right) \\
u^{*}\left(e^{i \theta}\right)=\sum_{i=2}^{n} a_{i} \theta^{i}+O\left(|\theta|^{n} \omega_{1}(|\theta|)\right)
\end{array}\right.
$$

where $\omega_{1}(|\theta|)=|\theta| \log 3 \pi /|\theta|+\omega_{0}(|\theta|)$ and the constants in the $O\left(|\theta|^{n} \omega_{1}(|\theta|)\right)$ terms depend only on $\Gamma$ and the constants $\left\{a_{i}\right\},\left\{b_{i}\right\},\left\{c_{i}\right\}$ are uniformly bounded by a constant depending only on $\Gamma$.

Proof. We have

$$
s(\theta)=\sum_{i=1}^{n-1} s^{(i)}(0) \frac{\theta^{i}}{i !}+O\left(|\theta|^{n} \log \frac{3 \pi}{|\theta|}\right)
$$

for $|\theta| \leqq \pi$. By the induction hypothesis, there exists a constant $K$ such that $\left|s^{(i)}(\theta)\right| \leqq K$ for $1 \leqq i \leqq n-1$ and $|\theta| \leqq \pi$, and such that 
the constant in the $O$ term is bounded by $K$. We also have

$$
V(s)=\sum_{i=2}^{n} V^{(i)}(0) \frac{s^{i}}{i !}+O\left(|s|^{n} \omega(s)\right)
$$

so that

$$
\begin{aligned}
v\left(e^{i \theta}\right)=V(s(\theta))= & \sum_{i=2}^{n} \frac{V^{(i)}(0)}{i !}\left[\sum_{j=1}^{n-1} s^{(j)}(0) \frac{\theta^{j}}{j !}+O\left(|\theta|^{n} \log \frac{3 \pi}{|\theta|}\right)\right]^{i} \\
& +O\left(\left[\sum_{j=1}^{n-1} s^{(j)}(0) \frac{\theta^{j}}{j !}+O\left(|\theta|^{n} \log \frac{3 \pi}{|\theta|}\right)\right]^{n} \omega_{0}(|\theta|)\right) \\
= & \sum_{i=2}^{n} b_{i} \theta^{i}+O\left(|\theta|^{n} \omega_{1}(|\theta|)\right) .
\end{aligned}
$$

The corresponding expression for $w\left(e^{i \theta}\right)$ is obtained similarly. Now, as in Lemma 8

$$
-u_{\theta}^{*}\left(e^{i \theta}\right)=v_{r}\left(e^{i \theta}\right) \frac{v_{\theta}\left(e^{i \theta}\right)}{u_{\theta}\left(e^{i \theta}\right)}+w_{r}\left(e^{i \theta}\right) \frac{w_{\theta}\left(e^{i \theta}\right)}{u_{\theta}\left(e^{i \theta}\right)}
$$

where $v_{\theta} / u_{\theta}=V_{s} / U_{s}$ for $|\theta|<\delta^{1}$. But $V_{s}(s) / U_{s}(s) \in C^{n-1, \omega}$ for $|\theta|<\delta$ so that

$$
\frac{V_{s}(s)}{U_{s}(s)}=\sum_{i=1}^{n-1} d_{i} s^{i}+O\left(|s|^{n-1} \omega(|s|)\right) \quad \text { for } \quad|\theta|<\delta
$$

and on rsing (10)

$$
\frac{v_{\theta}\left(e^{i \theta}\right)}{u_{\theta}\left(e^{i \theta}\right)}=\sum_{i=1}^{n-1} f_{i} \theta^{i}+O\left(|\theta|^{n-1} \omega_{1}(|\theta|)\right)
$$

Since $\Gamma \in C^{n-1,1}, v_{r}\left(e^{i \theta}\right) \in C^{n-2, \omega_{2}(t)}$ where $\omega_{2}(t)=K t(\log 3 \pi / t)$, so that

$$
\begin{aligned}
v_{r}\left(e^{i \theta}\right) \frac{v_{\theta}\left(e^{i \theta}\right)}{u_{\theta}\left(e^{i \theta}\right)}= & {\left[\sum_{i=0}^{n-2} g_{i} \theta^{i}+O\left(|\theta|^{n-1} \log \frac{3 \pi}{|\theta|}\right)\right] } \\
& \cdot\left[\sum_{i=1}^{n-1} f_{i} \theta^{i}+O\left(|\theta|^{n-1} \omega_{1}(|\theta|)\right)\right] \\
= & \sum_{i=1}^{n-1} h_{i} \theta^{i}+O\left(|\theta|^{n-1} \omega_{1}(|\theta|)\right) .
\end{aligned}
$$

A similar expansion holds for $w_{r}\left(e^{i \theta}\right) w_{\theta}\left(e^{i \theta}\right) / u_{\theta}\left(e^{i \theta}\right)$ so that

and

$$
u_{\vartheta}^{*}\left(e^{i \theta}\right)=\sum_{i=1}^{n-1} m_{i} \theta^{i}+O\left(|\theta|^{n-1} \omega_{1}(|\theta|)\right) \quad \text { for } \quad|\theta|<\delta
$$

$$
u^{*}\left(e^{i \theta}\right)=\sum_{i=2}^{n} a_{i} \theta^{i}+O\left(|\theta|^{n} \omega_{1}(|\theta|)\right) \text { for }|\theta| \leqq \pi .
$$

In each case the coefficients of the expansions and the constants in the $O$ terms are bounded uniformly, the bound depending only on $\Gamma$.

${ }^{1}$ At points $\theta_{0}$ where $d s / d \theta=0$ we mean by $v_{0}\left(e^{i \theta_{0}}\right) / u_{\theta}\left(e^{i \theta_{0}}\right)$ the limit as $\theta \rightarrow \theta_{0}$. 
Proof of Theorem 3. Let us first suppose that $\Gamma, F$ are as in Lemma 5. Then by Lemma 9, (9) holds. We may then apply lemma 2 to $i \lambda(z), \mu(z)$ and $\nu(z)$ to conclude that

$$
\begin{aligned}
& \left|\lambda^{(n+1)}(r)\right| \leqq M_{n} \int_{1-r}^{\pi} \frac{\omega_{1}(t)}{t^{2}} d t \quad(0<r<1) \\
& \left|\mu^{(n+1)}(r)\right| \leqq M_{n} \int_{1-r}^{\pi} \frac{\omega_{1}(t)}{t^{2}} d t
\end{aligned}
$$

and

$$
\left|\nu^{(n+1)}(r)\right| \leqq M_{n} \int_{1-r}^{\pi} \frac{\omega_{1}(t)}{t^{2}} d t
$$

Since the constants involved in (9) are bounded by a constant depending only on $\Gamma, M_{n}$ depends only on $\Gamma$. Thus, for all $|\theta| \leqq \pi$ we have

$$
\left|\lambda^{(n+1)}\left(r e^{i \theta}\right)\right| \leqq \sqrt{3} M_{n} \int_{1-r}^{\pi} \frac{\omega_{1}(t)}{t^{2}} d t
$$

and the corresponding inequalities obtain for $\mu$ and $\nu$.

Part (i) of the theorem then follows from Lemma 3, with $\omega_{1}$ rather than $\omega_{0}$.

Furthermore, by the corollary to Lemma 2, if $\Gamma$ is positioned as in Lemma 5 then there exists a constant $K$ depending only on $\Gamma$, such that $\left|\lambda^{(m)}(1)\right| \leqq K,\left|\mu^{(m)}(1)\right| \leqq K$ and $\left|\nu^{(m)}(1)\right| \leqq K$ for $m=1,2 \cdots, n$. By the equations (8) one sees that $\left|\lambda^{(m)}\left(e^{i \theta}\right)\right|,\left|\mu^{(m)}\left(e^{i \theta}\right)\right|$, and $\left|\nu^{(m)}\left(e^{i \theta}\right)\right|$ are bounded by $\sqrt{3} K$ for all $\theta$ and each $m, 1 \leqq m \leqq n$. From this it follows that $\left|s^{(n)}(\theta)\right|$ is bounded for all $\theta$ by a constant $c_{n}$ depending only on $\Gamma$.

We may now see that Lemma 9 and Theorem 3 are true with $\omega_{0}(|\theta|)$ in place of $\omega_{1}(\theta)$.

Since $s^{(n)}(\theta)$ is continuous and bounded, $s(\theta) \in C^{n-1,1}$ i.e.,

$$
s(\theta)=\sum_{i=1}^{n-1} s^{(i)}(0) \frac{\theta^{i}}{i !}+O\left(|\theta|^{n+1}\right)
$$

where the coefficients and the constant in the $O$ term are bounded by some constant $K$. Then, using (11) instead of (10) in the proof of Lemma 9, we obtain (9) with $\omega_{0}(|\theta|)$ instead of $\omega_{1}(|\theta|)$. Then Theorem 3 may be proved with $\omega_{0}(|\theta|)$ instead of $\omega_{1}(|\theta|)$.

\section{REFERENCES}

1. R. Courant, Dirichlet's Principle, Conformal Mapping, and Minimal Surfaces, Interscience, New York 1950.

2. G. Golusin, Geometrische Funktionentheorie, Deutsche Verlag Der Wissenschaften Berlin 1956. 
3. S. Hildebrandt, Boundary Behavior of Minimal Surfaces, Archive for Rational Mechanics and Analysis, 35 (1969), 47-82.

4. D. Kinderlehrer, The boundary regularity of minimal surfaces, Annali Sci. Norm. Sup. Pisa 23 (1969), 711-744.

5. H. Lewy, On the boundary behavior of minimal surfaces, Proc. Nat. Acad. Sci., 37 (1951), 103-110.

6. J. C. C. Nitsche, On new results in the theory of minimal surfaces, Bull. Amer. Math. Soc., 71 (1965), 195-270.

7. J. C. C. Nitsche, The boundary behavior of minimal surfaces, Kellogg's theorem and branch points on the boundary, Inventiones Math., 8 (1969), 313-333.

8. S. E. Warschawski, On differentiability at the boundary in conformal mapping, Proc. Amer. Math. Soc., 12 (1961), 614-620.

9. - On the higher derivatives at the boundary in conformal mapping, Trans. Amer. Math. Soc., 38, No. 2 (1935), 310-340.

10. - Boundary derivatives of minimal surfaces, Archive for Rational Mechanics and Analysis, 38 (1970), 241-256.

Received May 18, 1970. Research supported (in part) by U.S. Air Force Grant AFOSR-68-1514. This paper is based upon results of the author's doctoral thesis with Professor S. E. Warschawski which was presented to the Graduate Division of the University of California, San Diego.

University of California, SAN Diego

AND

San Diego State College 



\section{PACIFIC JOURNAL OF MATHEMATICS}

\section{EDITORS}

H. SAMELSON

Stanford University

Stanford, California 94305

C. R. Новву

University of Washington

Seattle, Washington 98105
J. DUGundJI

Department of Mathematics

University of Southern California

Los Angeles, California 90007

RICHARD ARENS

University of California

Los Angeles, California 90024

\section{ASSOCIATE EDITORS}
E. F. BECKENBACH
B. H. NeumanN
F. WOLF
K. YOSHIDA

\section{SUPPORTING INSTITUTIONS}
UNIVERSITY OF BRITISH COLUMBIA
CALIFORNIA INSTITUTE OF TECHNOLOGY
UNIVERSITY OF CALIFORNIA
MONTANA STATE UNIVERSITY
UNIVERSITY OF NEVADA
NEW MEXICO STATE UNIVERSITY
OREGON STATE UNIVERSITY
UNIVERSITY OF OREGON
OSAKA UNIVERSITY
UNIVERSITY OF SOUTHERN CALIFORNIA
STANFORD UNIVERSITY
UNIVERSITY OF TOKYO
UNIVERSITY OF UTAH
WASHINGTON STATE UNIVERSITY
UNIVERSITY OF WASHINGTON
AMERICAN MATHEMATICAL SOCIETY CHEVRON RESEARCH CORPORATION TRW SYSTEMS
NAVAL WEAPONS CENTER 


\section{Pacific Journal of Mathematics}

\section{Vol. 37, No. $1 \quad$ January, 1971}

Gregory Frank Bachelis and Haskell Paul Rosenthal, On unconditionally

converging series and biorthogonal systems in a Banach space .........

Richard William Beals, On spectral theory and scattering for elliptic

operators with singular potentials .........................

J. Lennart (John) Berggren, Solvable and supersolvable groups in which every element is conjugate to its inverse ........................ 21

Lindsay Nathan Childs, On covering spaces and Galois extensions ..........

William Jay Davis, David William Dean and Ivan Singer, Multipliers and

unconditional convergence of biorthogonal expansions..............

Leroy John Derr, Triangular matrices with the isoclinal property ............

Paul Erdős, Robert James McEliece and Herbert Taylor, Ramsey bounds for

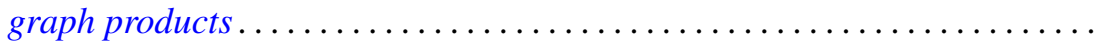

Edward Graham Evans, Jr., On epimorphisms to finitely generated

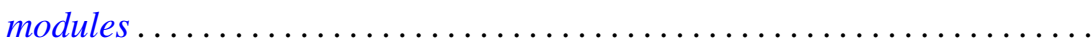

Hector O. Fattorini, The abstract Goursat problem ................. 51

Robert Dutton Fray and David Paul Roselle, Weighted lattice paths .........

Thomas L. Goulding and Augusto H. Ortiz, Structure of semiprime $(p, q)$

radicals ...........................................

E. W. Johnson and J. P. Lediaev, Structure of Noether lattices with join-principal maximal elements ....

David Samuel Kinderlehrer, The regularity of minimal surfaces defined over

slit domains

Alistair H. Lachlan, The transcendental rank of a theory. .

Frank David Lesley, Differentiability of minimal surfaces at the boundary ...

Wolfgang Liebert, Characterization of the endomorphism rings of divisible torsion modules and reduced complete torsion-free modules over complete discrete valuation rings....

Lawrence Carlton Moore, Strictly increasing Riesz norms.

Raymond Moos Redheffer, An inequality for the Hilbert transform ...

James Ted Rogers Jr., Mapping solenoids onto strongly self-entwined,

circle-like continua..........................

Sherman K. Stein, B-sets and planar maps ................... 217

Darrell R. Turnidge, Torsion theories and rings of quotients of Morita

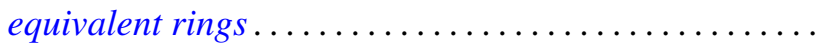

Fred Ustina, The Hausdorff means of double Fourier series and the principle of localization ................................

Stanley Joseph Wertheimer, Quasi-compactness and decompositions for arbitrary relations.

Howard Henry Wicke and John Mays Worrell Jr., On the open continuous images of paracompact $\check{C}$ ech complete spaces... 